\title{
Speedy validation sought for new cardiotoxicity testing strategy
}

On 23 July 2013, the US Food and Drug Administration (FDA), the Health and Environmental Sciences Institute (HESI) and the Cardiac Safety Research Consortium (CSRC) presented a new paradigm for cardiotoxicity testing, with a lofty goal of validating and standardizing the new assays in 2 years' time. A drastic change from current requirements, the proposal entails two non-clinical solutions: an in silico model that will probably integrate as many as six individual ion channel assays and electrophysiological tests on stem-cell-derived cardiomyocytes (Nature Rev. Drug Discov. 12, 565-567; 2013).

Current regulations include non-clinical and clinical tests - namely, the measurement of electrical current through the HERG (also known as $\mathrm{KCNH} 2$ ) potassium channel, an in vivo QT assay in an animal model and a trial examining the drug's effects on healthy volunteers' QT interval (a thorough QT study). Attendees of the meeting - 180 stakeholders from industry, regulatory agencies and academia — largely agreed that, because this current paradigm is costly and leads to false positives, a new approach could be more useful and predictive.

But whether the two proposed approaches should replace the current requirements, particularly the thorough QT study, was a point of debate in the meeting - at which many attendees were hearing about the assays for the first time. Their acceptance, of course, hinges on how well they perform in validation studies. But one message was clear: the industry is not ready to rely solely on these proposed approaches anytime soon.

"At this point, I hesitate to use these new tools for cardiovascular safety studies, because key gaps need to be addressed," says Hugo Vargas, Scientific Director, Safety and Exploratory Pharmacology at Amgen. "How closely do these [stem] cells resemble authentic cardiomyocytes, and can drug-induced changes in these cells be used to assess pro-arrhythmic risk? Likewise, how well do in silico [action potential duration] algorithms model cardiac electrophysiology, and response to drugs? The answers to these questions are critical." Indeed, Vargas notes that his direct experience with embryonic stem-cell-derived cardiomyocytes indicates that these cells are sensitive to HERG/IKr blockers, but not sodium channel $\mathrm{Na}_{\mathrm{v}} 1.5 / \mathrm{IKs}$ blockers, which would misinform a cardiac risk assessment ( . Pharmacol. Toxicol. Methods 68, 74-81; 2013).

The ultimate goal would be to replace the thorough QT study, which the FDA estimates has cost companies a total of US $\$ 1$ billion since current guidelines were established in 2005. However, Phase I studies will still be needed to evaluate the effects of new drugs on electrocardiogram intervals, atrioventricular conduction and heart rate, says meeting

It's safe to say that this is a potentially paradigm-shifting technology and I think most people are enthusiastic about it having positive health implications.
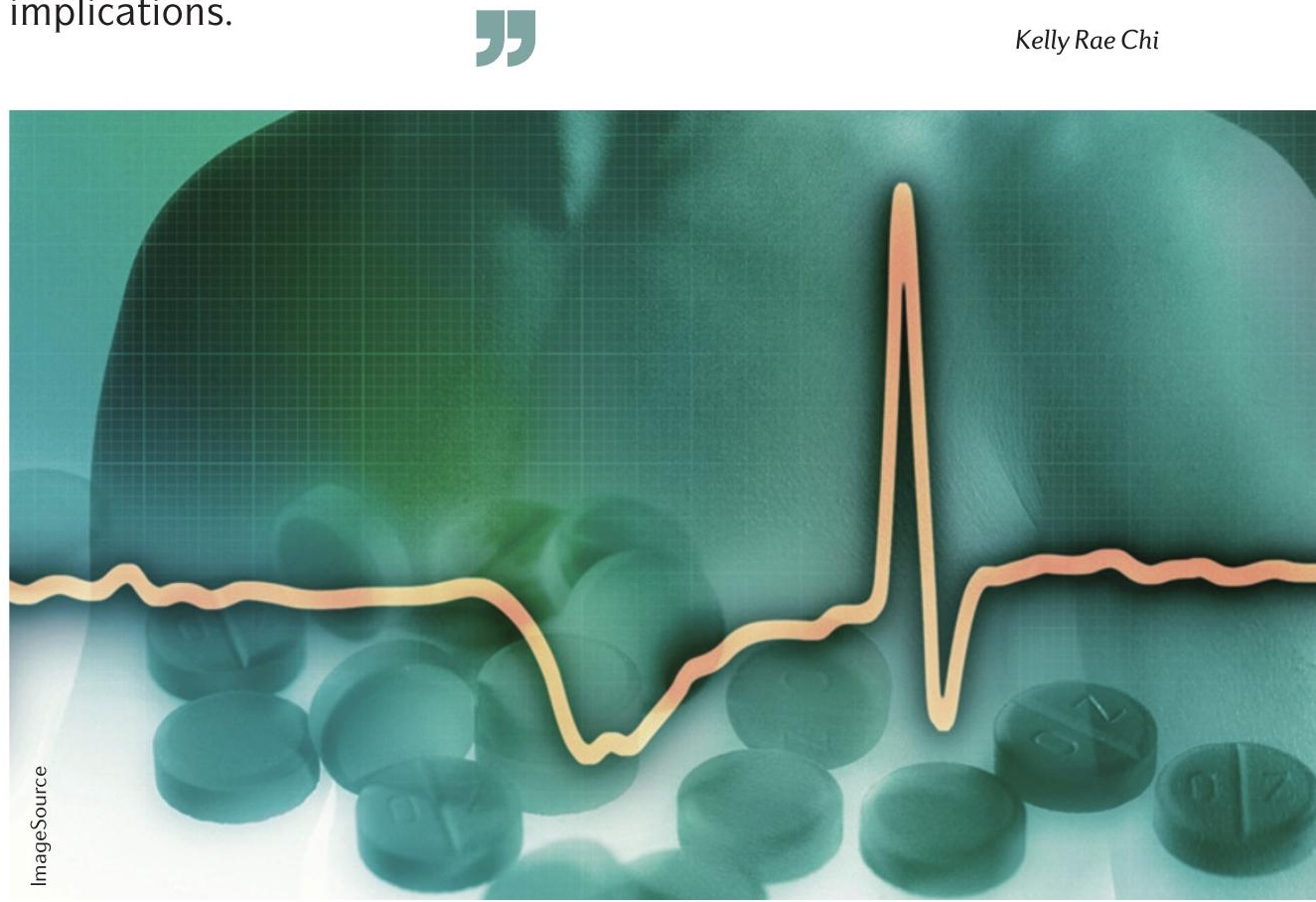

organizer Philip Sager, a consulting professor of medicine at Stanford University School of Medicine and Chair of the CSRC's Scientific Programs Committee. If findings arose that were not anticipated based on preclinical testing, that would cause concern.

The potential cost implications divided opinion among meeting attendees especially because they depend on big unknowns, such as whether sponsors would have enough confidence to forgo a thorough QT study. The costs also depend on how the assays are conducted and when in the pipeline they would be deployed: earlier steps would involve testing more compounds and greater costs.

The next steps still need defining. For example, says Vargas, "what is the scope of the validation effort? What companies can contribute resources to facilitate these studies? How much time will it take?". As for the number of compounds used in validation, discussions are focused on 10-20.

Validation and standardization will bring together many groups, including the HESI, the CSRC, the FDA, modelling experts, and probably others such as the Safety Pharmacology Society, who will need to first define a roadmap with multiple work-streams, says Sager.

“It's hard to say how it will play out. [But] it's safe to say that this is a potentially paradigm-shifting technology and I think most people are enthusiastic about it having positive health implications," says Andrew Erdman, Associate Head, Early Development Safety, Global Safety Risk Management at Genentech.

Kelly Rae Chi 